\title{
Late presenting Congenital Diaphragmatic Hernia Mimicking Complicated Pneumonia with? an Air Leak - A Case Report
}

Satam GH Alshammari*1, Saeed Rashid Alzahrani ${ }^{1}$, Tariq Jamal Alanezi ${ }^{2}$, Razan Ebrahim Alanfeesah ${ }^{3}$, Manar Jassem Alshammari ${ }^{3}$, Abdullah Al-Shamrani ${ }^{4}$

${ }^{1}$ Pediatric Pulmonologist, ${ }^{2}$ Pediatric Intensivist, ${ }^{3}$ Pediatric Department, Pediatric Department, Alyammamh Hospital, Riyadh, Saudi Arabia. ${ }^{4}$ Pediatric Pulmonologist and Sleep Physician,

Prince Sultan Medical Military City (PSMMC), Riyadh, Saudi Arabia

*Corresponding author: Satam GH Alshammari, Email: d_satam@icloud.com, Mobile: 009665506046664

\begin{abstract}
Background: Congenital diaphragmatic hernia $(\mathrm{CDH})$ is an anatomical defect of the diaphragm, which allows protrusion of abdominal viscera into the chest, causing serious pulmonary and cardiac complications in the neonate. Objective: In this case report we aimed to present a rare case of late presentation of congenital diaphragmatic hernia. Patient and method: We reported a late diagnosis of $\mathrm{CDH}$ at age of 3 months, who was referred to our center as a persistent left sided chest infection that needs necessarily intervention.

Result: At this late-presenting patient, which could be due to small defect in the diaphragm and could be missed antenatally or even in early postnatal period, it is expected to get worse when bowel distended, or patient stressed. Management of this condition was explored in detail, survival and prognostic factors were addressed.

Conclusion: $\mathrm{CDHs}$ are still a diagnostic challenge. Some are still missing in our community, and we need to improve medical services starting from antenatal visit. $\mathrm{CDH}$ should be kept in mind as a potential differential diagnosis in early infancy period for any patient presented with respiratory distress.
\end{abstract}

Keywords: CDH, Pneumonia, Post neonatal, Postnatal, Respiratory distress.

\section{INTRODUCTION}

Congenital diaphragmatic hernia $(\mathrm{CDH})$ is a birth defect from diaphragm. Usually suspected during neonatal period, when newborn presents with respiratory distress. Very few case reports are available in literature for its late presentation. Due to marked improvement in the antenatal service majorities of the cases are diagnosed antenatally and referred for inutero transfer to appropriate tertiary center to improve the survival ${ }^{(\mathbf{1})}$.

Aim of the present case report was to increase the awareness about presentation of mild or missed congenital lung disease, and to explore some areas of medical services that need improvement.

\section{CASE REPORT}

Patient information: We present a case of 3month-old girl who was admitted through the Emergency Department with a history of bluish discoloration of the face, shortness of breath for 1 day before admission. She was a full-term, with uneventful pregnancy, ultrasonography was done at $29^{\text {th }}$ weeks of pregnancy and was unremarkable with history of polyhydramnios in the last month of gestational age. The baby was delivered through vertex vaginal delivery and had a birth weight of $2.6 \mathrm{~kg}$. There was no respiratory distress soon after delivery.

The baby was discharged on the $2^{\text {nd }}$ day in good condition. She was the first baby to young nonconsanguineous parents. The baby was predominantly on bottle milk and was nursed as well by mother and there was no concern for her in the first 3 months regarding both feeding and breathing. At the age of 3 months, the baby was admitted to Shaqraa Hospital (in the northwest of the capital, Riyadh, Saudi Arabia) due to empyema following chest infection. The patient was presented there with a septic picture in form of severe respiratory distress, cyanosis, and hypotension. The chest X-ray showed left sided cystic lesion and pneumothorax. She was treated with needle and chest tube inserted then transferred to Alyammamh Hospital at Riyadh region, as a life-threatening case for further management. The parents denied history of fever, nasal congestion, eye discharge, skin rash or abnormal movement. The diagnosis of empyema was made based on clinical and radiological finding. She was started on ceftriaxone and vancomycin.

The examination on arrival to the Emergency Department at Alyammamh Hospital revealed an alert baby with all growth parameters within normal percentile. The weight: $7 \mathrm{~kg}(90 \%)$ Length: $59 \mathrm{~cm}$ (50\%) Head circumference: $40 \mathrm{~cm}(50 \%)$. She was afebrile, with a moderate respiratory distress in which the respiratory rate was 55/minute, tachycardiac with heart rate of $140 /$ minute, blood pressure was maintained at $75 / 43 \mathrm{mmHg}$ and her oxygen saturation was lowish $89 \%$ in room air, she required $2 \mathrm{~L} /$ minute of nasal cannula oxygen to maintain a saturation of greater than $93 \%$.

She had chest tube in the left side with a minimal drain connected to negative suction of $10 \mathrm{~cm}$ of water. On auscultation there was diminished breath sounds on the left side, with no additional sounds. Apart from 
tachycardia, the cardiovascular system examination was within normal limits. Abdomen was without deformity, soft, lax, and no organomegaly. She was alert with good tone and reflexes, and the rest of examination was normal.

\section{Investigations}

The blood gas analysis showed partially compensated respiratory alkalosis $\left(\mathrm{pH} 7.48, \mathrm{PCO}_{2} 26\right.$ mmHg and $\mathrm{HCO}_{3} 19.4$ meq/l). A complete blood count, hemoglobin level $(11.4 \mathrm{~g} / \mathrm{dl})$, the white blood cell count $\left(8.410^{9} / \mathrm{L}\right)$ and platelet count $\left(46310^{9} / \mathrm{L}\right)$ were both normal. Serum electrolyte levels were within normal; sodium was $143 \mathrm{mmol} / \mathrm{L}$ and potassium was $3.7 \mathrm{mmol} / \mathrm{L}$.

Coagulation profile was prolonged; blood culture was negative. The patient's chest X-ray in our center was misleading initially as large cystic lesion with a pressure effect.

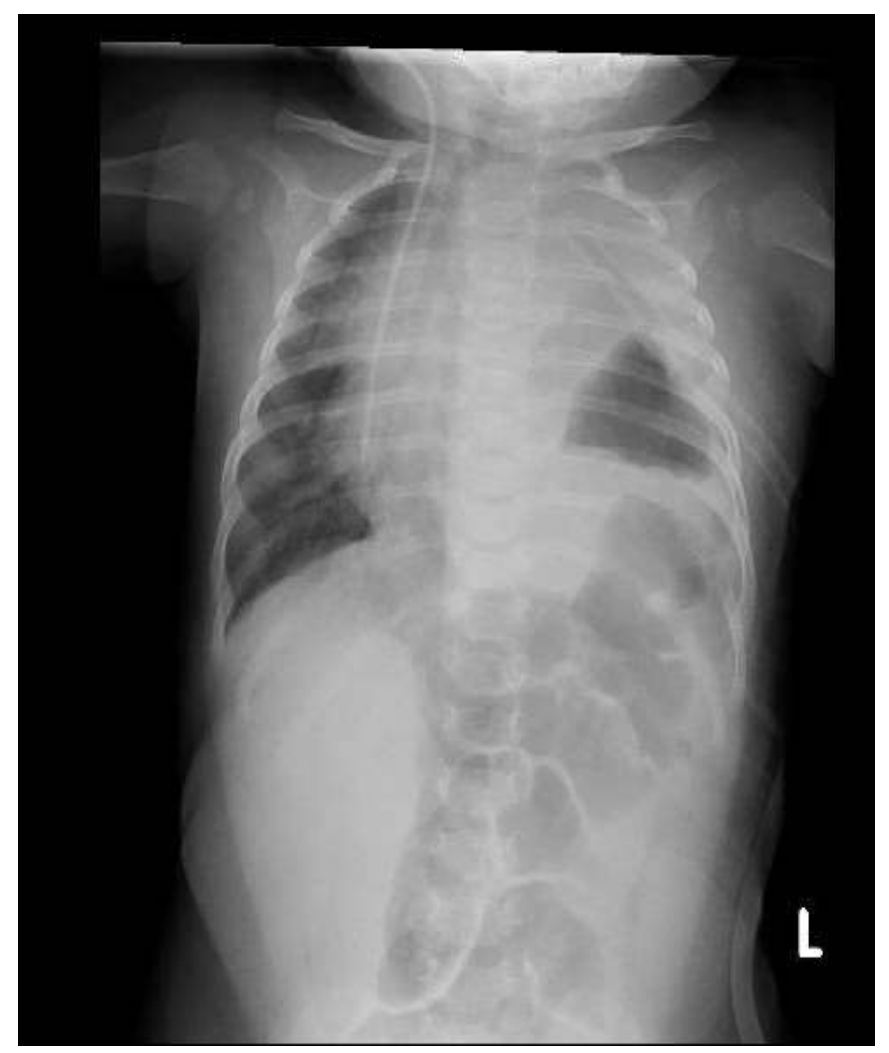

Figure (1): Anterior-posterior (AP) film, showed large cystic shadow on left sided chest, raised hemi diaphragm but difficult to assess the continuity, left sided chest tube in-situ, marked collapse of the left upper zone, deviation of the trachea and heart to the right sided with a pressure effect on left sided lung, secondary hyperinflation of the right lung, and central line in right ventricle.

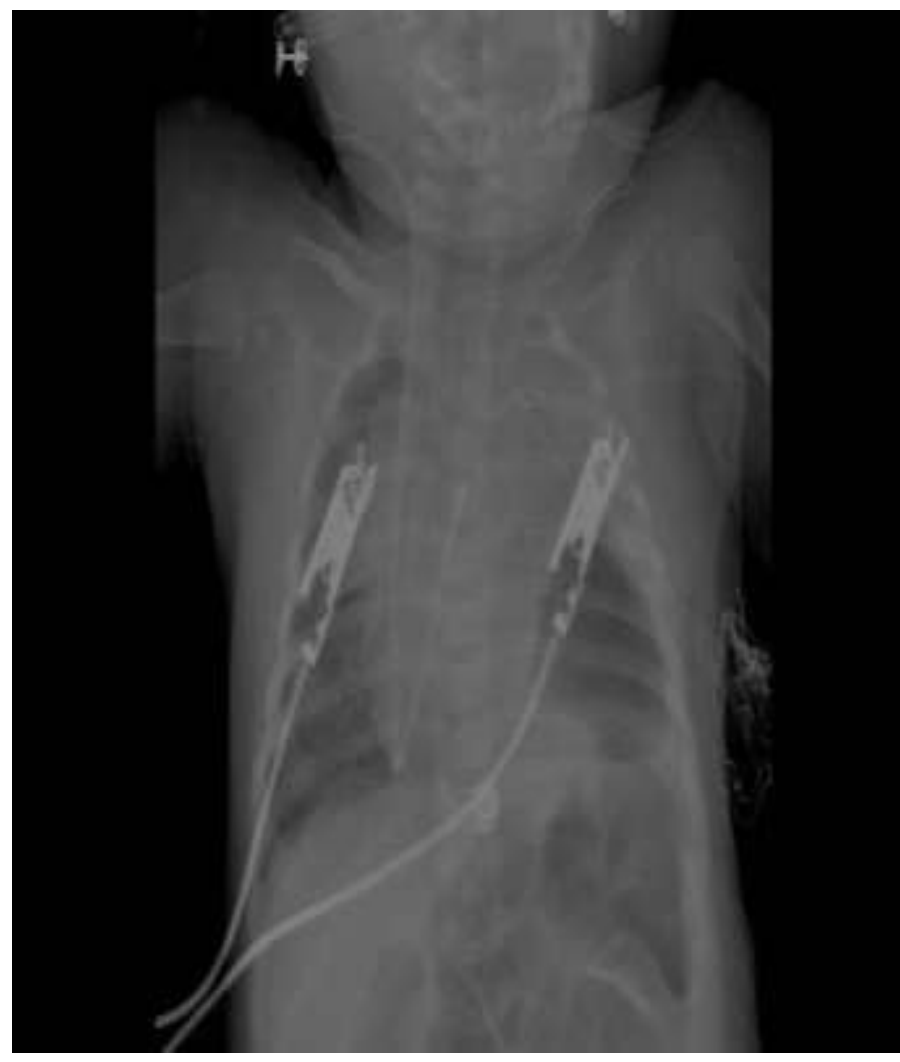

Figure (2): Preoperative AP film, large defect in the posterior lateral part of the left sided diaphragm with a continuation of radiolucent area extended to left sided hemi thorax (could be gastric or bowel), collapse of the left upper zone, significant deviation of the mediastinum, very advance central line, wires of ECG leads, very thick pleura and potential insignificant pleural effusion further, the X-ray is a typical of large Bochdalek hernia.

Chest Computed tomography: showed cystic air in the left lung lower lobe connected to the bowel (transverse colon) passing though diaphragm advising for fluoroscopy.

Chest ultrasound: showed mild insignificant pleural effusion.

Since the patient continued to have a moderate respiratory distress, she was shifted to the intensive care for respiratory support and the patient was labelled as a diaphragmatic hernia with potential clinical sepsis and chest infection, patient was supported with fluid, oxygen and double antibiotic (cefotaxime and vancomycin), surgeon was involved when the acute phase and coagulation improved then the patient was taken for surgical correction with a smooth postoperative course. 


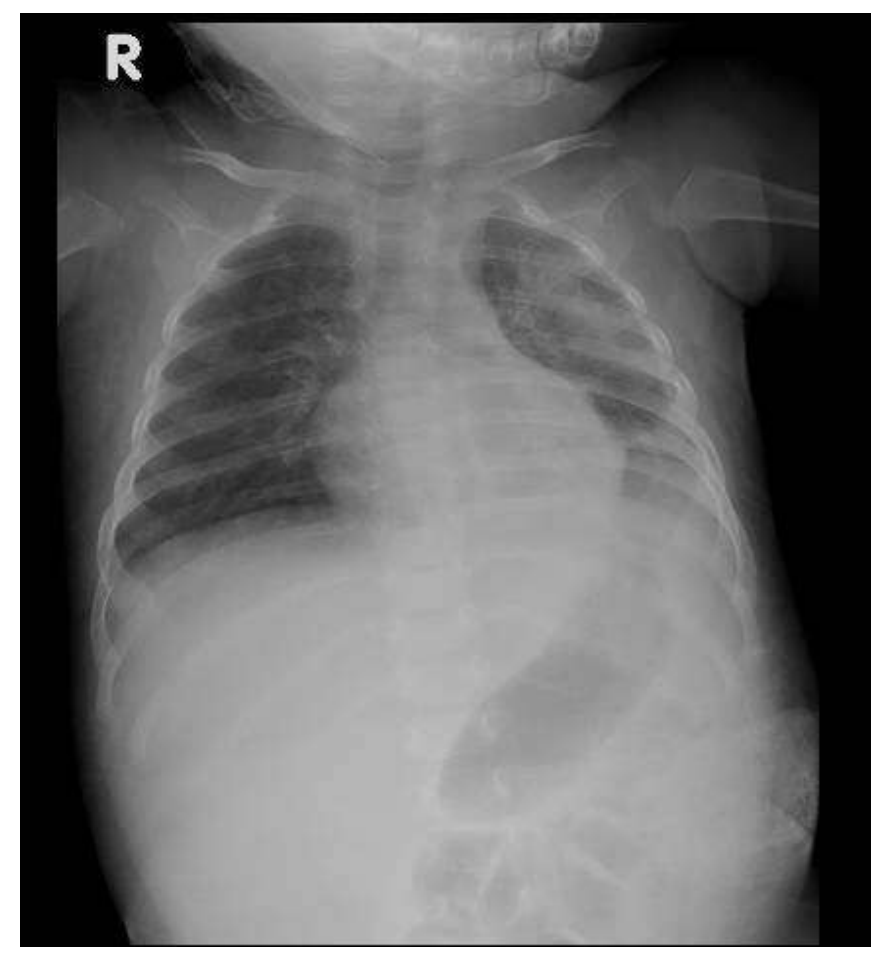

Figure (3): Postoperative AP film: marked improvement in the aeration of the left sided lung, mediastinum back to normal, still some evidence of opacity and segmental or subsegmental atelectasis on left side.

\section{DISCUSSION}

A congenital diaphragmatic hernia is a rare but potentially fatal anomaly, accounting for $8 \%$ of all congenital anomalies, with an incidence of 1 in 2,500 births ${ }^{(1)}$. It is characterized by the herniation of the abdominal organs into the pleural cavity, thus compressing the lungs and displacing the mediastinum to the contralateral side. Binomial hypoplasia and persistent pulmonary hypertension remain major mortality factors. The definition of an intrathoracic stomach is a condition in which at least two-thirds of the stomach opens into the posterior mediastinum, and it can be the result of a developmental defect, including $\mathrm{CDH}$, congenital hiatal hernia, congenital esophageal insufficiency, and asymmetric mucosa in the lower part of the esophagus ${ }^{(2)}$. The patient always suffers from respiratory distress in the neonatal period with a small number of limited differential diagnoses ${ }^{(3)}$.

The literature indicates that children with right-sided $\mathrm{CDH}$ present chronic symptoms in $57.4 \%$ of cases, while left-sided $\mathrm{CDH}$ appears predominantly with severe symptoms, which is also the common anatomical type of $\mathrm{CDH}$. In our case, $\mathrm{CDH}$ was in the left side, which is still rare in the rare late presentation of $\mathrm{CDH}{ }^{(3)}$. The diagnosis of a congenital diaphragmatic hernia usually occurs before birth, as ascites of amniotic fluid can be an early sign. Our patient has a history of excess fluid but there were no signs of shortness of breath in the early newborn period. There was no serial ultra sound antenatally and mother was not diabetic as a common cause of polyhydramnios. In about $5-25 \%$ of cases are late presenting, after the neonatal period ${ }^{(\mathbf{1})}$.

In this period it mainly depends on clinical suspicion and chest $x$-rays, although the size of the hernia and the amount of digestive herniation may cause confusion in the interpretation of the chest X-rays and may confuse the picture that shows the differential diagnosis of pneumonia, tumor, pneumothorax, and the diaphragm event as it happens to our patient when it was mistakenly described as a cystic lung lesion and due to persistent sepsis and chest infection in a narrowed chest tube it was inserted on the left side to relieve the degree of respiratory distress. Fortunately, the sticky left side of the chest was uninfected or perforated. During the physical examination, a bumped abdomen is very suspicious due to a hiatal hernia, which was not as evident in our cases as it is often the case in the postpartum ward with the distressed patient. Displaced heart sounds, respiratory failure, and chest bowel sounds are other supportive clinical findings. During the radiography, sac-like structures may be observed that are half-full of the chest (intestine), the mediastinum and the abdomen relatively free of gas. A chest X-ray is the most common way to diagnose $\mathrm{CDH}$.

If $\mathrm{CDH}$ is suspected, ultrasound may be helpful, with further diagnostic imaging including computed tomography (CT), magnetic resonance imaging (MRI), or contrast studies of the upper and lower GI tract ${ }^{(4,5)}$.

Treatment in severe cases and prenatal diagnosis is recommended as an immediate intubation soon after delivery in order to perform immediate decompression of the chest with abdominal straps, preventing pulmonary hypertension. All newborns must be intubated immediately after birth or upon diagnosis, with a nasogastric tube inserted on continuous aspiration. We must be careful with assisted ventilation in order to maintain low breathing pressure, to avoid damage or fracture of the corresponding lung. Surgical repair should be performed only after fixation, through an incision of the abdomen under the rib while reducing the contents of the hernia in the abdominal cavity and closing the diaphragmatic defect ${ }^{(6)}$.

As a late-presenting $\mathrm{CDH}$ can be a challenging diagnosis but the prognosis of late presenting $\mathrm{CDH}$ patients is usually better than in those cases which were diagnosed prenatally or shortly after birth, because the management of these patients is different and easier. Survival is between $97-100 \%$, in comparison to neonatal $\mathrm{CDH}$, with patients having uncomplicated recovery and few postoperative complications ${ }^{(7)}$. Finally, like our patient, those patients with latepresentation have relatively small defect carries a much better prognosis than large defects particularly those large enough to necessitate repair with a Gore-Tex patch $^{(8)}$. Our patient has a smooth postoperative course, 
echo heart was normal with evidence of pulmonary hypertension and patient tolerated feeds well and discharged with normalizing saturation with a follow up in the pediatric clinic for observation of long-term complication like gastroesophageal reflux disease, scoliosis, or recurrent hernia. Fortunately this case was not suffering from neurological effect or learning disability.

\section{CONCLUSION}

$\mathrm{CDH}$ is a therapeutic challenge. Although, $\mathrm{CDH}$ can be diagnosed antenatally by ultrasonography but few cases can be missed or presented late either in infancy or even late childhood. Pediatrician should include congenial lung malformation in the differential diagnosis for children presented with chest infection in these periods. Utilization of expert should be encouraged especially pediatric pulmonologist. Input from radiologist can be very helpful and crucial; for this we recommend that regular radiological activities with pediatrician should be encouraged. CT is very helpful for detailed anatomical description of the lung if the diagnosis is still obscured. Finally ultrasonography is an operator dependent in which it can be very informative in an expert hand. The case explores certain areas that need some improvement whether clinical bed side management or through interpretation of the images that been conducted for this patient. Surgical outcome is excellent despite some difficulties in confirming and managing this patient.

Conflicts of interest: We declare that there is no conflict of interest regarding the publication of this article.

\section{No funding was obtained or needed.}

Author's contribution: All authors read and gave final approval for this manuscript.

\section{Ethic approval:}

A signed written consent was taken from the mother of this child for participation and publication of medical details. Confidentiality of patient's data was ensured at all stages. The authors declare that ethics approval was not required for this case report.

\section{REFERENCES}

1. Topor L, Pătrăncuş T, Caragața R et al. (2015): "Left congenital diaphragmatic hernia-Case report. Chir (Bucur), 110 (1): 84-7.

2. Xia B, Hong C, Tang J et al. (2017): Congenital diaphragmatic hernia in association with congenital short esophagus: A case report. Medicine (Baltimore), 96(51): 8996.

3. Garg P, Bansal R, Dagla $\mathrm{R}$ et al. (2018): Congenital diaphragmatic hernia-A case report. JMSCR., 6 (9):12731.

4. Maciej B (2004): Late-presenting congenital diaphragmatic hernia in children: a clinical spectrum. Pediatric Surgery International, 20: 658-669.

5. Kim J, Couluris M, Schnapf B (2011): Latepresenting left-sided Morgagni congenital diaphragmatic hernia in a 9-Year-Old Male. Pulmonology, 11:4-9.

6. Lava J, Hettwer G, Reginatto C et al. (2012): Congenital diaphragmatic Bochdaleck hernia: case report. International Archives of Medicine, 5(30):1-4.

7. Blackstone $\mathbf{M}$, Mistry $\mathbf{R}$ (2007): Late-presenting congenital diaphragmatic hernia mimicking bronchiolitis. Pediatric Emergency Care, 23(9):653-656.

8. Rouse C, Schmidt L, Brock L et al. (2017): Case report congenital diaphragmatic hernia presenting in a 7-dayold infant. Emergency Medicine, 3: 1-3. 